INTERNATIONAL JOURNAL OF RESEARCHES IN BIOSCIENCES, AGRICULTURE \& TECHNOLOGY (C) VISHWASHANTI MULTIPURPOSE SOCIETY (Global Peace Multipurpose Society) R. No. MH-659/13(N)

www.vmsindia.org

\title{
BUTTERFLY DIVERSITY IN RELATION TO A RELATIVE ABUNDANCE AND STATUS IN SELOO CITY, WARDHA MAHARASHTRA, CENTRAL INDIA
}

\begin{abstract}
:-
A survey was conducted to record the butterfly diversity and the status and occurrence of butterfly species in and around Seloo city, Central India from 2011 to 2018. A total of 91 species of butterflies belonging to Papilionidae (07 species), Pieridae (14 species), Nymphalidae (29 species), Lycaenidae (28 species), Hesperiidae (12 species) and 01 species was recorded from the Riodinidae. Of all the total 91 species, $29 \%$ were commonly occurring, $46 \%$ were very common, $9 \%$ were not rare, $13 \%$ were rare and $3 \%$ were very rarely occurring. About 06 species of the recorded ones come under the protection category of the Indian Wild Life protection Act 1972. The observations support the value of the Seloo city area in providing valuable resources for butterflies.
\end{abstract}

Ashish Tiple

Dept of Zoology, Vidhyabharti College, Seloo, Wardha, Maharshtra- 442 104, India. E-mail: ashishdtiple@yahoo.co.in

Keywords: India, Lepidoptera, diversity, Seloo city, Maharashtra

\section{INTRODUCTION}

Butterflies are the most beautiful and colourful creatures on the earth and have a great aesthetic value. Butterflies have always been a subject of interest and they are probably next only to birds in their universal popularity evoking curiosity and fondness among children, naturalists and scientist alike. This is partly attributable to the great variety and beauty of their colour patterns and partly to their aromatic transformation during mimicry and migration (Kunte, 2000). They constitute one of the most important links in ecological pyramids of food chain i.e. a link between plants and other predators like birds, reptiles and spiders; transforming and transmitting energy from green plants to the animal. Amongst the invertebrates, butterflies are becoming sufficiently well studied for them to be used for general conservation planning in some parts of the tropics as a representative insect group (Thomas, 1992).

Butterflies are very sensitive biota to environment and are directly affected by changes in the habitats, atmospheric temperature and the weather conditions; they can be good indicators of environment changes (Tiple et al., 2006). Most of the butterflies are seasonal in their occurrence, they are abundant only from beginning of monsoon (June-July) till the early winter (August-November) and decline in species abundance from late winter
(December-January) up to the end of summer (Tiple and Khurad 2009).

Butterflies have been studied systematically since the early $18^{\text {th }}$ century and about 19,238 species are documented worldwide by 1998 (Heppner, 1998). This figure is not constant because of the continuous addition of new butterflies and also due to ongoing disagreements between taxonomists over the status of many species.

The Indian subcontinent a diverse terrain, climate and vegetation hosts about 1,504 species of butterflies (Tiple 2011) of which Peninsular India hosts 351, and the Western Ghats 336. In Central India, the butterfly diversity was reported earlier by Forsayeth, (1884); Swinhoe, (1886); Betham, $(1890,1891)$ \& Witt, (1909). D'Abreeu, (1931) documented a total of 177 species occurring in the erstwhile Central Provinces (now Madhya Pradesh and Vidarbha). In the recent past, several workers have studied butterflies from urban, rural and protected areas of Vidarbha. 65 species belonging to 52 genera representing 7 families from Pench Tiger Reserve, (Maharashtra) (Sharma \& Radhakrishnan, 2005); 68 species of butterflies of 50 genera were recorded from Tadoba Andhari Tiger Reserve (Sharma \& Radhakrishnan, 2006) and 103 species of butterflies were recorded from Melghat Tiger Reserve (Wadatkar, 2008). Tiple \& Khurad, (2009) reported 145 species of butterflies recorded, of which 62 species were new records 
for Nagpur city. Recently, Tiple, 2010 documented 111 species of butterflies in Tadoba National Park.

The present study was started with a view to examine the diversity of butterfly from Seloo city. Since there is no published checklist of butterfly from Seloo city prior to this, the present work can form the baseline for further research.

\section{MATERIAL AND METHOD}

The Seloo city (20083'73”N; 78070'70”E; $265 \mathrm{~m})$ is situated nearer to Bor Wildlife Sanctuary at the bank of river Bor along with the dense shrub, natural vegetation and tree vegetation which are the major attraction to the butterflies. It has tropical wet and dry climate with dry conditions, an annual rainfall of about 1,205 $\mathrm{mm}$ (June to September); temperature raises up to $48.9^{\circ} \mathrm{C}$ during summer (MarchJune) and falls up to $10^{\circ} \mathrm{C}$ to $6.9^{\circ} \mathrm{C}$ in winter (November-January). Annual relative humidity varies in between $22 \%$ to $80 \%$ (Tiple 2011).

The findings presented here are based on field surveys carried out on weekly basis from 2011 to 2018 at in and around Seloo city. Butterfly were surveyed in Seloo city and surrounding areas. Identification of the butterflies was primarily made directly in the field. In critical condition specimens were collected only with handheld aerial sweep nets and subsequently released without harm. Each specimen was placed in plastic bottles and carried them to the laboratory for further identification with the help of field guide (Wynter-Blyth, 1957; Kunte, 2000). The observed butterflies were grouped in five categories on the basis of number of sighting in the field. The butterflies were categorized as VCVery common (> 100 sightings), C- Common (51100 sightings), NR- Not rare (16-50 sightings), R- Rare (2-15 sightings), VR- Very rare $1<2$ sightings) (Tiple and Khurad 2009 ).

\section{RESULTS AND DISCUSSION}

During the course of study 91 species of butterflies belonging to 6 families, were recorded. The highest number of butterflies belongs to the family Nymphalidae (29 species). Followed by the Lycaenidae 28 species, 14 species to Pieridae, 12 species to Hesperiidae, 07 species to Papilionidae and 01 species was recorded from the Riodinidae.

Among the 112 species of butterflies about $29 \%$ (26) were occurring common, 46\%
(42) species were very common, 9\% (08) were not rare, $13 \%$ (12) were rare, and 3\% (03) were very rare (i.e. Graphium nomius, Lxias pyrene, Jamides bochus). The observed and identified species, their status in and around of Seloo city are listed in Table 1.

Among the 91 butterflies recorded, 05 species come under the protection category of the Indian Wild Life (protection) Act 1972 (Tiple, 2011; Gupta \& Mondal, 2005) (i.e. Pachliopta hector, Euploea core, Hypolimnas misippus, Euchrysops cnejus, Lampides boeticus). As reported by Kunte (2000), an objective revision of the scheduled list is necessary in providing appropriate and adequate legal protection to Indian butterflies.

In India we have two peak seasons, March-April and October-November for butterfly abundance (Wynter-Blyth (1957). The abundances of diverse species were positively affected by approaching summer, high relative humidity and more rainfall. In the present investigation most butterfly species were observed from the monsoon (hot/wet season) to early winter (cool/wet season) but subsequently declined in early summer (March). Among the 91 species of butterflies, Papilio demoleus, Pachliopta aristolochiae, Catopsilia pomona, Eurema hecabe, Danaus chrysippus, Tirumala limniace, Acraea violae, Euploea core, Junonia lemonias, Catochrysops strabo, Chilades putli were occurred throughout the year (JanuaryDecember), whereas remaining 80 species of butterflies were prominently observed only after June-July till the beginning of summer (AprilMay). Increasing species abundance from beginning of monsoon (June-July) till the early winter (August-November) and decline in species abundance from late winter (January February) up to the end of summer (Fig. 1) have also been reported by Tiple et al. (2007); Tiple and Khurad (2009) and Tiple 2012 in similar climatic conditions in this region of Central India.

The findings of the present study underline the importance of city as a preferred habitat for butterflies. If the landscaping and maintenance of gardens are carefully planned, the diversity of butterflies may increase in Seloo city providing a rich ground for butterfly conservation as well as for research. This study will also add to our future attempts in understanding the complex nature of 
mutualistic interaction between butterflies and flowering plants that is essential for continuity of ecosystem services. The present list of butterfly species is not conclusive and exhaustive and future exploration will be continued to update this checklist.

\section{REFERENCES}

1. Betham J. A. 1890. The butterflies of the Central Provinces. Journal of the Bombay Natural History Society, 5: 19-28; 151-161; 279-286.

2. Betham J. A. 1891. The butterflies of the Central Provinces. Journal of the Bombay Natural History Society, 6: 175-183; 318-331.

3. D'Abreeu E. A., 1931. The central provinces butterfly list. Records of the Nagpur museum number VII. Government printing city press, 39pp.

4. Forsayeth R.W., 1884. Life history of sixty species of Lepidoptera observed in Mhow, Central India. Transactions of the Entomological Society of London, 3: 377-419.

5. Gupta I. J. \& Mondal D. K., 2005. Red Data Book, Part II: Buttrflies of India. Zoological Society of India, Kolkata, pp. 535.

6. Heppner J. 1998. Classification of Lepidoptera. Part I Introduction. Holarctic Lepidoptera. 5 (1): 148.

7. Kunte, K., 2000. Butterflies of Peninsular India. Universities Press (Hyderabad) and Indian Academy of Sciences (Bangalore).

8. Sharma R. M. \& Radhakrishnan C., 2005. Insecta: Lepidoptera (Phopalocera and Grypocera). Fauna of Pench National Park, Conservation area series. Zoological Survey of India, 20: 251-274.

9. Sharma R. M. \& Radhakrishnan C., 2006. Insecta: Lepidoptera (Phopalocera and Grypocera). Fauna of Todoba Andhari Tiger Reserve, Conservation area series. Zoological Survey of India, 25: $255-277$.

10. Swinhoe C., 1886. On the Lepidoptera of Mhow. Proceedings of Zoological Society of London pp.421-465.

11. Thomas C. D. 1992. Habitat use and geographic ranges of butterflies from the wet lowlands of Costa Rica. Biological Conservation. 55: 269281.

12. Tiple A. D., \& Khurad A. M., 2009. Butterfly Species Diversity, Habitats and Seasonal
Distribution in and around Nagpur City, Central India. World Journal of Zoology, 4(3): 153-162.

13. Tiple A. D., 2010. Butterfly Fauna of Tadoba National Park and Surroundings, Chandrapur, Maharashtra (Central India). Hislopia, 3(1):1-9.

14. Tiple A. D., 2011. Butterflies of Vidarbha region, Maharashtra State, central India. Journal of Threatened Taxa, 3(1): 1469-1477.

15. Tiple A.D. 2012. Butterfly species diversity, relative abundance and status in Tropical Forest Research Institute, Jabalpur, Madhya Pradesh, central India. Journal of Threatened Taxa. 4(7): 2713-2717.

16. Tiple A. D., Khurad A. M. \& Dennis R. L. H., 2007. Butterfly diversity in relation to a humanimpact gradient on an Indian university campus. Nota Lepidopteralogica, 30 (1): 179-188.

17. Tiple A. D., Deshmukh V. P. \& Dennis R. L. H., 2006. Factors influencing nectar plant resource visits by butterflies on a university campus: implications for conservation. Nota Lepidopteralogica, 28: 213-224.

18. Wadatkar J. S., 2008. Butterflies of Melghat Tiger Reserve, Maharashtra with notes on their abundance, status. Mel-Vyaghra XIII (III). 1-5.

19. Witt D.O., 1909. The butterflies (Rhopalocera) of the Nimar district, Central Provinces. Journal of the Bombay Natural History Society, 19(3):564571 .

20. Wynther-Blyth M. A., 1957. Butterflies of the Indian Region. Bombay Natural History Society, pp. 523.

Figure 1. The variations of species composition throughout the year in Seloo city

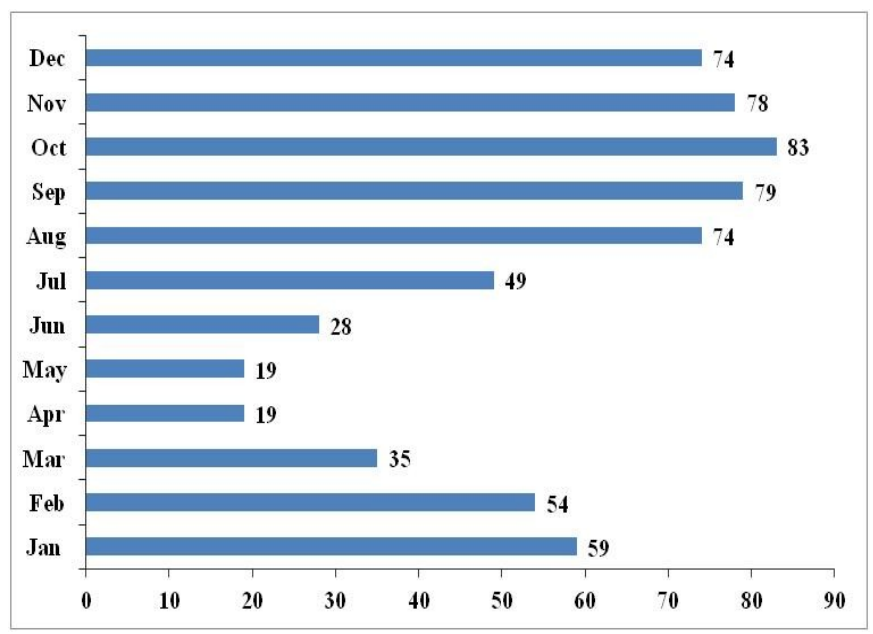


Table 1. List of butterflies recorded from Seloo city together with common name, status and Occurrence.

\begin{tabular}{|c|c|c|c|c|}
\hline $\begin{array}{l}\text { Sr. } \\
\text { No. }\end{array}$ & Scientific Name & Common Name & $\begin{array}{c}\text { Statu } \\
\mathbf{s}\end{array}$ & $\begin{array}{c}\text { Occurren } \\
\text { ce } \\
\text { (months) }\end{array}$ \\
\hline & Papilionidae (07) & & & \\
\hline 1. & Graphium agamemnon (Linnaeus, 1758) & Tailed Jay & $\mathrm{C}$ & $8-1$ \\
\hline 2. & Graphium doson (C. \& R. Felder, 1864) & Common Jay & NR & $8-1$ \\
\hline 3. & Graphium nomius (Esper, 1799) & Spot Swordtail & VR & $3-7$ \\
\hline 4. & Pachliopta aristolochiae (Fabricius, 1775) & Common Rose & $\mathrm{C}$ & $7-2$ \\
\hline 5. & Pachliopta hector (Linnaeus, 1758) & Crimson Rose & $\mathrm{C}$ & $7-2$ \\
\hline 6. & Papilio demoleus Linnaeus, 1758 & Lime Butterfly & $\mathrm{VC}$ & $1-12$ \\
\hline \multirow[t]{2}{*}{7.} & Papilio polytes Linnaeus, 1758 & Common Mormon & $\mathrm{VC}$ & $7-2$ \\
\hline & Pieridae (14) & & & \\
\hline 8. & Belenois aurota (Fabricius, 1793) & Pioneer & $\mathrm{VC}$ & $9-2$ \\
\hline 9. & Catopsilia pomona (Fabricius, 1775) & Common Emigrant & $\mathrm{VC}$ & $1-12$ \\
\hline 10. & Catopsilia pyranthe (Linnaeus, 1758) & Mottled Emigrant & $\mathrm{VC}$ & $7-2$ \\
\hline 11. & Cepora nerissa (Fabricius, 1775) & Common Gull & $\mathrm{VC}$ & $1-12$ \\
\hline 12. & Colotis danae (Fabricius, 1775) & Crimson Tip & $\mathrm{VC}$ & $6-10$ \\
\hline 13. & Colotis etrida (Boisduval, 1836) & Small Orange Tip & NR & $8-12$ \\
\hline 14. & Ixias pyrene Linnaeus, 1764 & Yellow Orange Tip & VR & $8-9$ \\
\hline 15. & Delias eucharis (Drury, 1773) & Common Jezebel & $\mathrm{VC}$ & $7-2$ \\
\hline 16. & Eurema brigitta(Stoll, [1780]) & Small Grass Yellow & $\mathrm{C}$ & $1-12$ \\
\hline 17. & Eurema hecabe (Linnaeus, 1758) & Common Grass Yellow & $\mathrm{VC}$ & $1-12$ \\
\hline 18. & Eurema laeta (Boisduval, 1836) & Spotless Grass Yellow & $\mathrm{VC}$ & $7-12$ \\
\hline 19. & Ixias marianne (Cramer, [1779]) & White Orange Tip & $\mathrm{C}$ & $8-11$ \\
\hline 20. & Leptosia nina (Fabricius, 1793) & Psyche & $\mathrm{R}$ & $8-1$ \\
\hline \multirow[t]{2}{*}{21.} & Pareronia hippie (Cramer, [1776]) & Common Wanderer & $\mathrm{C}$ & $8-2$ \\
\hline & Nymphalidae (29) & & & \\
\hline 22. & Acraea violae (Fabricius, 1793) & Tawny Coster & $\mathrm{VC}$ & $10-12$ \\
\hline 23. & Ariadne ariadne (Linnaeus, 1763) & Angled Castor & $\mathrm{VC}$ & $9-2$ \\
\hline 24. & Ariadne merione (Cramer, [1777]) & Common Castor & $\mathrm{C}$ & $10-2$ \\
\hline 25. & Byblia ilithyia (Drury, [1773]) & Joker & $\mathrm{C}$ & $6-12$ \\
\hline 26. & Charaxes solon (Fabricius, 1793) & Black Rajah & $\mathrm{R}$ & $10-2$ \\
\hline 27. & Danaus chrysippus (Linnaeus, 1758) & Plain Tiger & $\mathrm{VC}$ & $1-12$ \\
\hline 28. & Danaus genutia (Cramer, [1779]) & Striped Tiger & $\mathrm{C}$ & $10-6$ \\
\hline 29. & Euploea core (Cramer, [1780]) & Common Indian Crow & $\mathrm{VC}$ & $1-12$ \\
\hline 30. & Euthalia aconthea Cramer, 1777 & Common Baron & $\mathrm{R}$ & $8-11$ \\
\hline 31. & Hypolimnas bolina (Linnaeus, 1758) & Great Eggfly & $\mathrm{C}$ & $6-3$ \\
\hline 32. & Hypolimnas misippus (Linnaeus, 1764) & Danaid Eggfly & $\mathrm{C}$ & $1-12$ \\
\hline 33. & Junonia almana (Linnaeus, 1758) & Peacock Pansy & $\mathrm{VC}$ & $6-2$ \\
\hline 34. & Junonia atlites (Linnaeus, 1763) & Grey Pansy & $\mathrm{VC}$ & $7-2$ \\
\hline 35. & Junonia hierta (Fabricius, 1798) & Yellow Pansy & NR & $8-2$ \\
\hline 36. & Junonia iphita (Cramer, [1779]) & Chocolate Pansy & $\mathrm{VC}$ & $8-3$ \\
\hline 37. & Junonia lemonias (Linnaeus, 1758) & Lemon Pansy & $\mathrm{VC}$ & $1-12$ \\
\hline 38. & Junonia orithya (Linnaeus, 1758) & Blue Pansy & $\mathrm{VC}$ & $10-4$ \\
\hline 39. & Melanitis leda (Linnaeus, 1758) & Common Evening Brown & $\mathrm{VC}$ & $1-12$ \\
\hline 40. & Moduza procris (Cramer, [1777]) & Commander & $\mathrm{C}$ & $8-1$ \\
\hline 41. & Mycalesis mineus (Linnaeus, 1758) & Dark Branded Bushbrown & $\mathrm{R}$ & $8-3$ \\
\hline 42. & Mycalesis perseus (Fabricius, 1775) & Common Bushbrown & $\mathrm{VC}$ & $7-3$ \\
\hline 43. & Phaedyma columella (Cramer, [1780]) & Short-banded Sailer & $\mathrm{R}$ & $9-11$ \\
\hline 44. & Neptis hylas (Linnaeus, 1758) & Common Sailer & $\mathrm{VC}$ & $7-3$ \\
\hline 45. & Phalanta phalantha (Drury, [1773]) & Common Leopard & $\mathrm{VC}$ & $6-3$ \\
\hline 46. & Symphaedra nais (Forster, 1771) & Baronet & $\mathrm{C}$ & $10-3$ \\
\hline 47. & Tirumala limniace (Cramer, [1775]) & Blue Tiger & $\mathrm{VC}$ & $1-12$ \\
\hline 48. & Ypthima asterope (Klug, 1832) & Common Threering & $\mathrm{C}$ & $7-9$ \\
\hline 49. & Ypthima huebneri (Kirby, 1871) & Common Fourring & $\mathrm{R}$ & $11-12$ \\
\hline
\end{tabular}




\begin{tabular}{|c|c|c|c|c|}
\hline 50. & Ypthima inica (Hewitson, 1865) & Lesser Threering & $\mathrm{C}$ & $9-12$ \\
\hline & Riodinidae (1) & & & \\
\hline 51. & Abisara bifasciata Moore, 1877 & Double-banded Judy & $\mathrm{R}$ & $8-10$ \\
\hline & Lycaenidae (28) & & & \\
\hline 52. & Acytolepis puspa (Horsfield, [1828]) & Common Hedge Blue & $\mathrm{VC}$ & $9-2$ \\
\hline 53. & Amblypodia anita Hewitson, 1862 & Leaf Blue & NR & $8-9$ \\
\hline 54. & Azanus jesous (Guérin-Méneville, 1849) & African Babul Blue & $\mathrm{C}$ & $10-2$ \\
\hline 55. & Azanus ubaldus (Stoll, [1782]) & Bright Babul Blue & $\mathrm{R}$ & $11-2$ \\
\hline 56. & Castalius rosimon (Fabricius, 1775) & Common Pierrot & VC & $1-12$ \\
\hline 57. & Catochrysops strabo (Fabricius, 1793) & Forget-Me-Not & $\mathrm{VC}$ & $1-12$ \\
\hline 58. & Chilades lajus (Stoll, [1780]) & Lime Blue & $\mathrm{VC}$ & $8-12$ \\
\hline 59. & Luthrodes pandava (Horsfield, [1829]) & Plains Cupid & $\mathrm{VC}$ & $1-12$ \\
\hline 60. & Chilades parrhasius (Fabricius, 1793) & Small Cupid & $\mathrm{R}$ & $7-2$ \\
\hline 61. & Freyeria putli (Kollar, [1844]) & Eastern Grass Jewel & $\mathrm{C}$ & $7-12$ \\
\hline 62. & Virachola isocrates (Fabricius, 1793) & Common Guava Blue & NR & $8-10$ \\
\hline 63. & Euchrysops cnejus (Fabricius, 1798) & Gram Blue & $\mathrm{VC}$ & $6-3$ \\
\hline 64. & Jamides bochus (Stoll, [1782]) & Dark Cerulean & VR & $7-2$ \\
\hline 65. & Jamides celeno (Cramer, [1775]) & Common Cerulean & $\mathrm{VC}$ & $7-3$ \\
\hline 66. & Lampides boeticus (Linnaeus, 1767) & Pea Blue & $\mathrm{VC}$ & $8-3$ \\
\hline 67. & Leptotes plinius (Fabricius, 1793) & Zebra Blue & VC & $7-3$ \\
\hline 68. & Prosotas nora (Felder, 1860$)$ & Common Lineblue & $\mathrm{C}$ & $7-3$ \\
\hline 69. & Psuedozizeeria maha (Kollar, [1844]) & Pale Grass Blue & $\mathrm{C}$ & $8-3$ \\
\hline 70. & Rapala iarbus (Fabricius, 1787) & Common Red Flash & $\mathrm{C}$ & $8-12$ \\
\hline 71. & Spindasis ictis (Hewitson, 1865) & Common Shot Silverline & NR & $6-8$ \\
\hline 72. & Spindasis schistacea (Moore, [1881]) & Plumbeous Silverline & $\mathrm{C}$ & $7-8$ \\
\hline 73. & Spindasis vulcanus (Fabricius, 1775) & Common Silverline & $\mathrm{VC}$ & $8-2$ \\
\hline 74. & $\begin{array}{l}\text { Tarucus balkanicus nigra Bethune-Baker, } \\
{[1918]}\end{array}$ & Black-spotted Pierrot & $\mathrm{C}$ & $8-1$ \\
\hline 75. & Tarucus callinara Butler, 1886 & Spotted Pierrot & $\mathrm{C}$ & $8-2$ \\
\hline 76. & Tarucus nara (Kollar, 1848) & $\begin{array}{l}\text { Rounded Pierrot/ Striped } \\
\text { Pierrot }\end{array}$ & $\mathrm{VC}$ & $7-2$ \\
\hline 77. & Zizeeria karsandra (Moore, 1865) & Dark Grass Blue & $\mathrm{VC}$ & $1-12$ \\
\hline 78. & Zizina otis (Fabricius, 1787) & Lesser Grass Blue & $\mathrm{VC}$ & $6-3$ \\
\hline 79. & Zizula hylax (Fabricius, 1775) & Tiny Grass Blue & $\mathrm{VC}$ & $6-3$ \\
\hline & Hesperiidae (12) & & & \\
\hline 80. & Badamia exclamationis (Fabricius, 1775) & Brown Awl & $\mathrm{C}$ & $8-11$ \\
\hline 81. & Borbo bevani (Moore, 1878) & Bevan's Swift & $\mathrm{R}$ & $8-11$ \\
\hline 82. & Borbo cinnara (Wallace, 1866) & Rice Swift & $\mathrm{VC}$ & $1-12$ \\
\hline 83. & Celaenorrhinus leucocera (Kollar, [1844]) & Common Spotted Flat & $\mathrm{R}$ & $8-9$ \\
\hline 84. & Coladenia indrani (Moore, [1866]) & Tricolour Pied Flat & NR & $9-12$ \\
\hline 85. & Hasora badra (Moore, [1858]) & Common Awl & $\mathrm{C}$ & $7-9$ \\
\hline 86. & Hasora chromus (Cramer, [1780]) & Common Banded Awl & VC & $9-10$ \\
\hline 87. & Pelopidas mathias (Fabricius, 1798) & Small Branded Swift & $\mathrm{VC}$ & $7-12$ \\
\hline 88. & Spialia galba (Fabricius, 1793) & Indian Skipper & $\mathrm{R}$ & $8-3$ \\
\hline 89. & Suastus gremius (Fabricius, 1798) & Indian Palm Bob & NR & $7-12$ \\
\hline 90. & Telicota bambusae (Moore, 1878) & Dark Palm Dart & $\mathrm{VC}$ & $8-12$ \\
\hline 91. & Telicota colon (Fabricius, 1775) & Pale Palm Dart & $\mathrm{C}$ & $7-10$ \\
\hline
\end{tabular}

